\title{
Kinematic Features of Unrestrained Vertical Arm Movements ${ }^{1}$
}

\author{
CHRISTOPHER G. ATKESON AND JOHN M. HOLLERBACH ${ }^{2}$ \\ Artificial Intelligence Laboratory and Department of Psychology, Massachusetts Institute of Technology, \\ Cambridge, Massachusetts 02139
}

\begin{abstract}
Unrestrained human arm trajectories between point targets have been investigated using a three-dimensional tracking apparatus, the Selspot system. Movements were executed between different points in a vertical plane under varying conditions of speed and hand-held load. In contrast to past results which emphasized the straightness of hand paths, movement regions were discovered in which the hand paths were curved. All movements, whether curved or straight, showed an invariant tangential velocity profile when normalized for speed and distance. The velocity profile invariance with speed and load is interpreted in terms of simplification of the underlying arm dynamics, extending the results of Hollerbach and Flash (Hollerbach, J. M., and T. Flash (1982) Biol. Cybern. 44: 67-77).
\end{abstract}

We have investigated unrestrained human arm trajectories between point targets using a three-dimensional tracking apparatus, the Selspot system. Our studies indicate the importance of examining natural unrestricted movements, as our results agree only in part with previous studies of arm movement. Past observations on multijoint human arm trajectories obtained from restricted horizontal planar movements measured with a gripped pantograph have shown in both humans and monkeys that point-to-point trajectories are essentially straight with bell-shaped velocity profiles (Morasso, 1981; Abend et al., 1982). Moreover, they satisfy a time scaling property that may be related to the underlying dynamics (Hollerbach and Flash, 1982). We sought to corroborate these observations for more natural unrestricted arm movements and also to examine the effects of different loads and of gravity on the arm trajectories. Our research on load effects has also led to the discovery of scaling laws for arm loads.

Path shape. A strategy for gaining insight into planning and control processes of the motor system is to look for kinematic invariances in trajectories of movement. The significance of straight-line movements of the hand during arm trajectories is that they imply movement planning at the hand or object level (Morasso, 1981; Holler-

Received June 26, 1984; Revised Tebruary 4, 1985;

Accepted March 13, 1985

\footnotetext{
1 I his paper describes research done at the Department of Psychology and at the Artificial intelligence Laboratory of the Massachusetts Institute of Technology. Support for this research was provided by National Institutes of Health Research Grant AM 26710, awarded by the National Institute of Arthritis, Metabolism, and Digestive Diseases, and by a National Science Foundation graduate fellowship (C. G. A.). We also acknowledge early contributions of Michael Propp and Jonathan Delatizky toward development of our Selspot system, and of Eric Saund for display software development.

${ }^{2}$ To whom correspondence should be addressed.
}

bach, 1982), that is to say, in terms of coordinates or variables that are external to the biological system and that could be matched to tasks or outside constraints.

If movements were planned in terms of joint variables, one would expect curved hand paths. The observed straight-line hand paths would seem to preclude this possibility (Morasso, 1981), yet in a series of papers examining unrestrained vertical arm movement (Soechting and Lacquaniti, 1981; Lacquaniti and Soechting, 1982; Lacquaniti et al., 1982), the hand trajectories were evidently straight at the same time that the joint rate ratio of shoulder and elbow tended toward a constant. This apparently contradictory situation of straight lines in both hand space and joint space has nevertheless been resolved recently in favor of hand space straight lines due to an artifact of two-joint kinematics near the workspace boundary (Hollerbach and Atkeson, 1984).

When hand movements are curved in response to task requirements or to internal control, it is not as clear what the planning variables are. For handwriting movements, Hollerbach (1981) proposed orthogonal task coordinates in the writing plane that yielded cursive script through coupled oscillation and modulation. Viviani and Terzuolo (1982) proposed hand variable planning for drawing as well as writing through proportional control of tangential velocity and radius of curvature. Morasso (1983) examined three-dimensional curved motion and proposed independent control of the curvature and torsion of the hand cartesian coordinates. Again arguing for joint-level planning but also for actuator-level planning, Soechting and Lacquaniti (1983) investigated curved movements resulting from change of target location during two-joint arm movement, and inferred both a linear relation between elbow and shoulder accelerations and stereotypical muscle electromyogram activity.

Time profile. In addition to the path of the arm, the other aspect of a trajectory is the time sequence along the path. This tangential velocity profile may through its shape also give insight into movement planning strategies. For motions under low spatiotemporal accuracy constraints, a common observation is a symmetrical and unimodal velocity profile. Crossman and Goodeve (1983) characterized these profiles as Gaussian for two different single degree of freedom movements: a pen-tapping movement constrained by a measurement wire and wrist rotation about the forearm axis. More recently, Hogan (1984) modeled the velocity profiles for single-joint elbow movement as fourth-order polynomials derived from a minimum-jerk cost function. In examining optimization criteria for single-joint movement, Nelson (1983) deduced that a minimum-jerk velocity profile is almost indistinguishable from simple harmonic motion for repelitive movement. Stein et al. (1985) modeled muscle activation and energetics for a single degree of freedom point-to-point movement, and showed that muscle force rise time or minimum energy yields a velocity profile very similar to minimum jerk.

The previous experiments involved single degree of freedom movement, either with one joint or an apparatus with one degree of freedom, for which the only independent parameter is the time dependence. Nevertheless, similar results have been found for multi- 
joint movement. In a relatively unconstrained pen movement to a trontal target like dart throwing, Beggs and Howarth (1972) observed a symmetrical sigmoid position trace. For horizontal two-joint arm movement measured with a planar pantograph, a number of researchers have noted a unimodal velocity profile essentially similar to the single-joint case (Morasso, 1981; Abend et al., 1982). Flash (1983) showed that this two-joint case is also describable by a minimum-jerk profile when movement is modeled in terms of cartesian coordinates of a mass moving in a plane. In addition. Flash (1983) showed that jerk minimization could predict the bimodal velocity profiles of curved movements observed in a task of obstacle avoidance or via point intersection (Abend et al., 1982). These results were interpreted as further evidence for hand-level planning.

When there are strict spatial accuracy constraints, the trajectories evidently take on new features. Often the velocity profile is segmented into several submovements: a coarse positioning submovement that brings the hand close to the target, and onc or more finc positioning submovements to reach the target (Annet et al., 1958; Langolf et al., 1976; Jagacinski et al., 1980; Carlton, 1980; Soechting, 1984). One model of the movement segmentation has been an iterative corrections model (Keele, 1968; Crossman and Goodeve, 1983) which, however, is now discounted (Langolf et al., 1976; Jagacinski et al., 1978; Carlton, 1980). At other times, researchers have found a unimodal velocity profile where the deceleration takes longer than acceleration, as in the single degree of freedom joystick positioning task of Taylor and Birmingham (1948). They also noted that peak velocity and acceleration varied linearly with movement distance, which implies a velocity profile invariance for this task. Crossman and Goodeve (1983) noted longer decelerations for a pen-tapping task, and Carlton (1980) noted them for a discrete pen movement task.

For conditions of temporal accuracy constraints, the speed/ accuracy relation seems to be linear rather than logarithmic as for Fitts' law, but the decelerations are also evidently longer than the accelerations (Schmidt et al., 1984). Schmidt et al. (1979) and Meyer et al. (1982) proposed different impulse variability models as alternatives to Fitts' law; the latter model is notable for the derivation of a symmetric acceleration profile with a double inflection point from acceleration to deceleration. The data of Schmidt et al. (1984), however, seem to call into doubt current formulations based on impulse variability.

Loads, gravity, and dynamics. The previous research has reported on the effect of kinematic constraints on trajectory formation. It is also important to examine the effect of external loads and gravity, since they modify the dynamics of movement and could conceivably alter trajectories in a way that gives insight into control processes. In one of the few studies in this area, Lacquaniti et al. (1982) report that trajectory is unaffected by a $2.5 \mathrm{~kg}$ load, in movements made under the effect of gravity. These results are presented as plots of elbow joint rate versus shoulder joint rate and do not pertain directly to the issue of tangential velocity profile invariance. Ruitenbeek (1984) reported velocity profile invariance for a single degree of freedom plunger movement under several programmed impedance loadings.

Most explanations for velocity profile shape and invariance deal with kinematic criteria, whether they be endpoint criteria or impulse variability. Impulse variability for single-joint movement is equivalent to a kinematic analysis because movement force and acceleration are proportional (neglecting viscosity): kinematics and dynamics are degenerate, and the levels of kinematic, dynamic, and actuational analysis are all equivalent. When multi-joint movement is considered, force and acceleration are no longer proportional but are related by inverse dynamics. Arbitrarily changing the time profile along a fixed path in order to change movement speed will change joint torques in a complicated manner (Hollerbach, 1984) and present a severe computational burden to the motor control system.

In contrast to kinematic criteria, Hollerbach and Flash (1982) proposed that an invariant velocity profile for a given path may have a dynamics underpinning. If movement speed along a fixed path is varied by stretching or compressing the velocity profile uniformly, then in principle the joint torques for the new movement speed are simply related to the old joint torques. This method of torque formation requires separate treatment of the gravity torques and the dynamic or drive torques, which include joint velocities and accelerations.

In demonstrating the possibility of speed scaling, Hollerbach and Flash (1982) examined only horizontal planar arm movements due to apparatus restrictions. Since gravity does not act in the horizontal plane of movement, a better test of speed scaling and the proposed separate treatment of gravity and drive torques is to investigate movement in a vertical plane where gravity is a factor. Recently we have developed the capability of measuring unrestricted vertical or any other movements with a Selspot system, and this paper reports in part on results concerning speed scaling for vertical arm movements.

Additionally, wo cxamine load effects on trajectory, where weights are held in a subject's hand. When examining load effects on movement, a cursory inspection of the dynamic equations would seem to indicate a complicated alteration of joint torques even for following the same trajectory. Speed changes and the presence of gravity evidently complicate the situation even further. If the trajectory is changed when loads are added, it would indicate that the motor system is unable to compensate for the load in order to realize desired movement kinematics. If the trajectory is unchanged, then somehow the motor system must have devised a strategy for compensating potentially complicated dynamic effects.

\section{Methods}

Unrestrained arm movements of subjects were measured with a Selspot system for self-paced, point-to-point reaching movements under varying conditions of target location, external load, and movement speed. Target zones were defined in a vertical sagittal plane, but arm movements were in no way restricted to conform to this plane except at the end points. In fact, it was noted that while the hand and wrist points stayed fairly well in the plane, nearly all subjects preferred to rotate the elbow out of the plane. Five right-handed, naive subjects participated in this study, two male and three female, in the age range of 25 to 40 .

\section{Experimental procedures}

Human subjects sat in a chair facing parallel to a vertical pegboard. Visible light-emitting diode (LED) targets were located on the ends of dowel pins projecting normally from the pegboard; this made it possible to define arbitrary three-dimensional positions of the targets. The targets were located in comfortable reach of subjects and in a vertical plane that permitted free swinging of the right arm close to the side of the body. Four target pairs were chosen as indicated in Figure 1. Only the two active targets were in place during the movement trials to avoid obstacles affecting the trajectories.

Subjects were instructed not to touch a target, but to move the tip of the first finger to a point 1 inch in front of the activated target LED. The subjects were told not to worry about the fine accuracy of the movement and were discouraged from making corrective movements after the initial movement ended. No accuracy constraints were placed on the movements.

Trials were conducted without visual feedback in a darkened room; light from the target LED illuminated a small volume and was lurned off under computer control as the subject's finger approached within $5 \mathrm{~cm}$ of the destination target zone. No instruction was given about the form of path or trajectory execution. In trials with an external load, the load was held by the subjects in their hand during the movement. Subjects were allowed to rest if the load or speed conditions were too taxing

Experimental protocol. The subject was familiarized with the locations of the target zones and the target sequence for a pair of targets. Trials were blocked first by target pair, then by load, and lastly by speed. (1) Target pairs were run in the sequence $1-4,3-7,2-6$, and $4-8$. (2) Three load conditions were run in the order no load, 2-lb. load, and either 3- or 4-1b. load depending on subject strength. (3) Three movement speeds were run corresponding to self-paced slow, normal, and fast speeds. The average durations of these movements were roughly 1200,800 , and $400 \mathrm{msec}$, respectively. (4) Six movements were executed between two targets, $A$ and $B$, by interspersing three movements from $A$ to $B$ with three movements from $B$ to $A$. There was one practice session of six movements only, for the target pair 1-4 under conditions of no load and slow speed. A movement trial, executed entirely under computer control, was conducted as follows. 


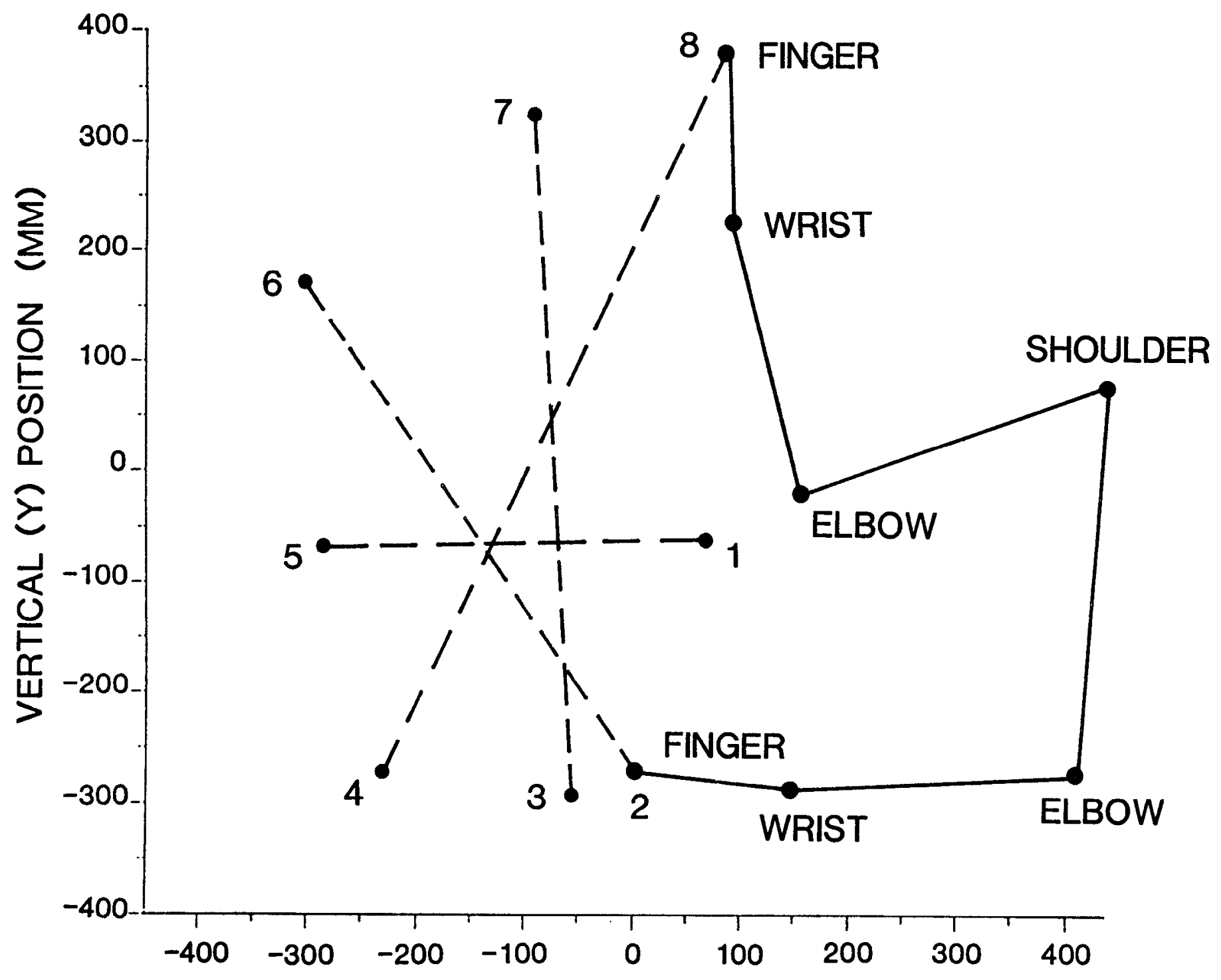

HORIZONTAL (X) POSITION (MM)

Figure 1. Location of targets and subject. The four pairs of targets used in this study are numbered 1-5, 2-6, 3-7, and 4-8. The locations of the targets shown here are approximate in that we adjusted the target locations for each subject to ensure that the targets were well within the subject's reach. The $X Y$ projections of the segments of a typical subject's arm are indicated in two configurations by the solid lines. Because the subject's elbow was often out of the $X Y$ plane by varying amounts, the lengths of the solid lines do not indicate the true arm segment lengths but only the lengths of their $X Y$ projections.

(1) An auditory cue was followed by illumination of the start target LED. (2) The subject positioned the index finger before the start target zone. (3) The start target LED was turned off and sampling was started. (4) The destination target LED was turned on 240 msec later, signaling the movement start. (5) As the subject's finger approached the target zone the destination target LED was furned off, leaving the subject in darkness. (6) The subject completed the movement.

Selspot system. The infrared I.ED markers were placed on the subject so as to facilitate later reconstruction of the subject's elbow and shoulder angles (to be presented in a subsequent paper). LED 1 was placed at the end of the subject's pointing finger. Two metal strips were prepared with an infrared marker at each end and attached to the subject's upper arm and forearm aligned with the axis of each body segment (Fig. 2); the wrist was marked approximately by one end of the forearm strip. Elbow and shoulder angles may be calculated from the three-dimensional coordinates of the strip LEDs.

The LEDs were each sampled at the maximum rate of $315 \mathrm{~Hz}$ by the Selspot system and the data were stored on a PDP 11/34 computer. The raw data consisted of $(X, Y)$ positions of LEDs on two camera detector plates. The cameras were approximately located at $90^{\circ}$ to each other and $45^{\circ}$ to the plane of movement and at a distance of $3 \mathrm{~m}$.

\section{Data processing}

The data were later processed off line to obtain the three-dimensional positions for the LED markers, joint angles, and other parameters of interest. The software for data processing evolved from previously developed aigorithms (Conati, 1977; Tetewsky, 1978). Currently our software is substantially different from other Selspot software, although we have incorporated some aspects of recent developments from Antonsson $(1978,1982)$ and Reece (1981).

Obtaining marker $L E D$ three-dimensional coordinates. The raw data were filtered with a fixed-length ( 63 points), finite impulse filter with no phase lag and characteristics of 0 to $6 \mathrm{~Hz}$ pass band and 6 to $24 \mathrm{~Hz}$ transition band (McClellan, et al., 1973). Special steps are required for bad LED data. (1) Data points are marked as bad in which (i) an LED was out of view or (ii) reflections of the infrared beam or other noise caused a spurious reading as detected by excess deviation from a regression applied to neighbors. (2) Bad points are filled in using a mixture of linear interpolation and cubic splines and the ends of the data record are extrapolated to allow filtering of all data. (3) The data are filtered, after which filled points are removed.

Data from each camera were then corrected by table lookup. These tables were produced by putting LED markers in known positions relative to each 


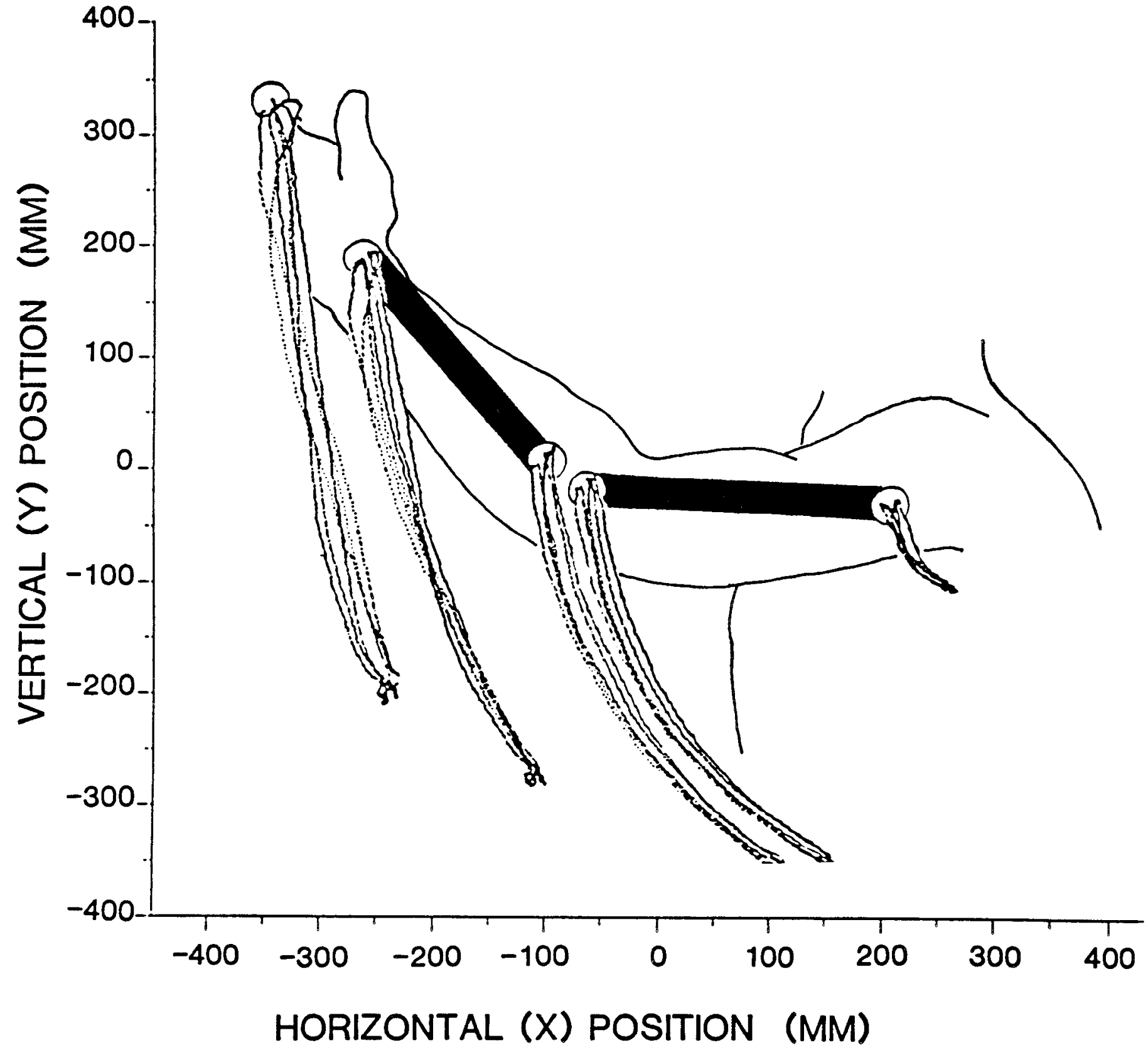

Figure 2. Attachment of Selspot markers and data presentation. Locations of the Selspot infrared LED markers and the typical format of the data presentation are shown. Note that the wrist and one of the elbow LEDs are connected by a rigid bar aligned with the forearm, and the shoulder and other elbow LEDs are similarly connected on a rigid bar aligned with the upper arm. The three-dimensional Selspot data are projected onto the $X Y$ plane. This projection shows most of the features of the path because these movements were almost planar (for the finger, wrist, and shoulder) and oriented parallel to the $X Y$ plane. In each data plot several movements are presented. Three upward movements (dotted lines) are indicated here by a dot at the location of the infrared LED for each sample (sampling frequency $315 \mathrm{~Hz}$ ). Three downward movements are indicated by solid lines marking the path of each infrared LED.

camera and recording the average measured positions. Deviations between expected and actual measurements were calculated and mapped into a 25 $\times 25$ correction table. Standard interpolation techniques were used to calculate the table originally and to read corrections from the table.

Three-dimensional positions of the LEDs were calculated from the corrected data using the known positions and orientations of the cameras and geometry. Points were marked as bad for which the vectors to the reconstructured LED position from each camera origin missed by greater than a certain threshold $(3 \mathrm{~cm})$, since with four parameters from the two cameras there is one redundant measurement. The Selspot system in our configuration can detect movements of the markers as small as $1 \mathrm{~mm}$. Currently, the absolute accuracy of the system is within $\pm 1 \mathrm{~cm}$.

Normalization of tangential velocity profiles. To check invariance of tangential velocity profile shape, movements must be normalized for time and distance. Define $v(t)$ as the experimental tangential velocity profile as a function of time $t, v_{\max }$ as the maximum tangential velocity in $v(t), d$ as the experimental movement distance, $v_{\text {ref }}$ as the reference velocity, and $d_{\text {ref }}$ as the reference distance. The reference velocity and distance are chosen arbitrarily, and all data records are scaled to them. Since the tangential velocity profiles $v(t)$ are almost always unimodal, the maximum tangential velocity $v_{\max }$ is well defined. Wo usc $v_{\max }$ rather than movement duration because of imprecision in determining movement start and stop points.

Now define time and distance scaling factors $c$ and $a$ as

$$
c=\frac{v_{\text {rel }}}{v_{\max }}, \quad a=\frac{d_{\text {ref }}}{d}
$$

The velocity profile $v^{\prime}(t)$ normalized first for distance is $v^{\prime}(t)=\operatorname{av}(t)$. The maximum velocity for the new velocity profile is then $v_{\max }^{\prime}=a v_{\max }$. Define a new time scaling factor $c^{\prime}=v_{\text {ref }} / v_{\max }^{\prime}=c / a$. Then the time-normalized velocity profile $v^{\prime \prime}(t)$ is 

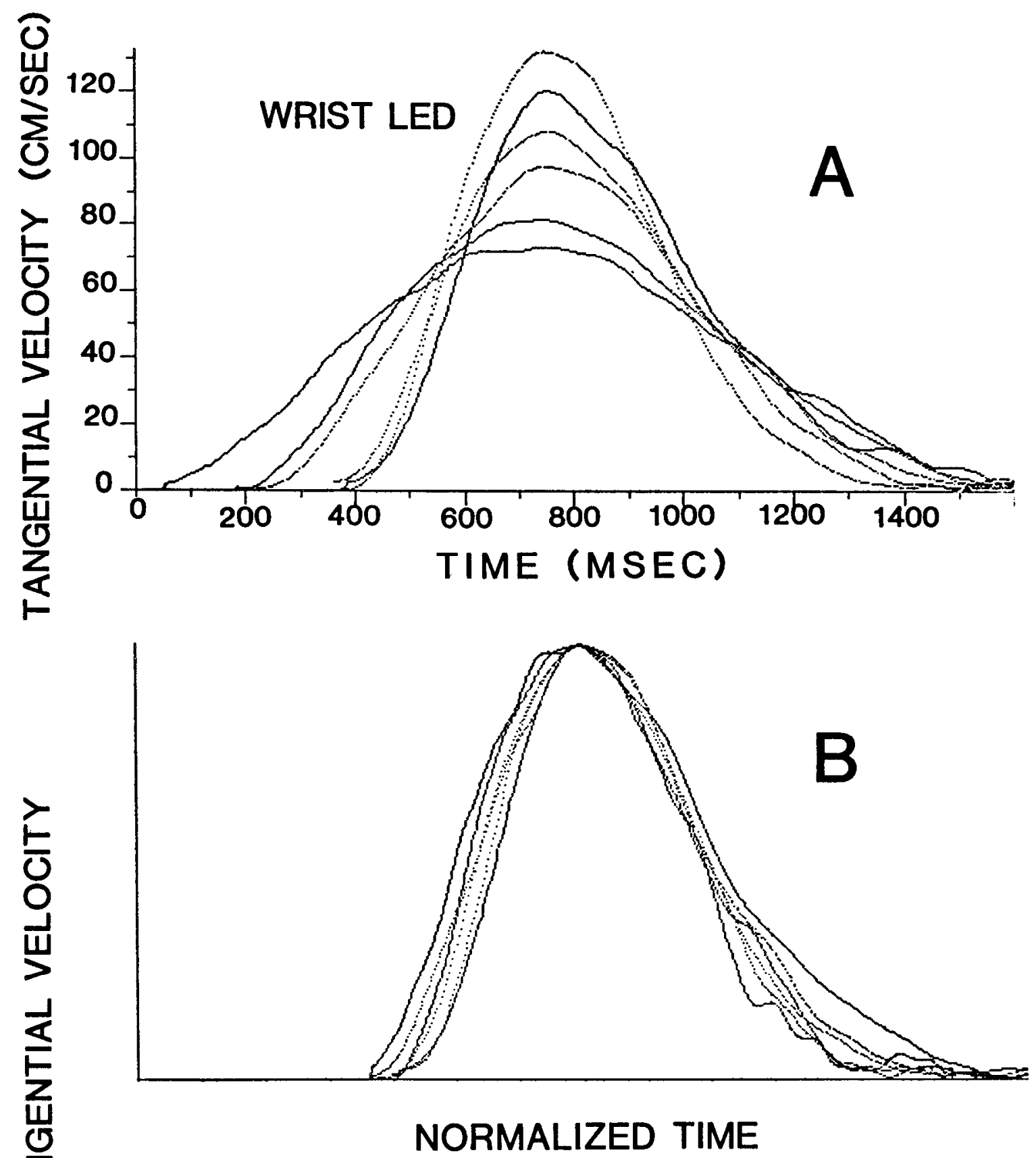

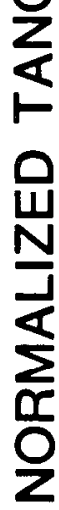

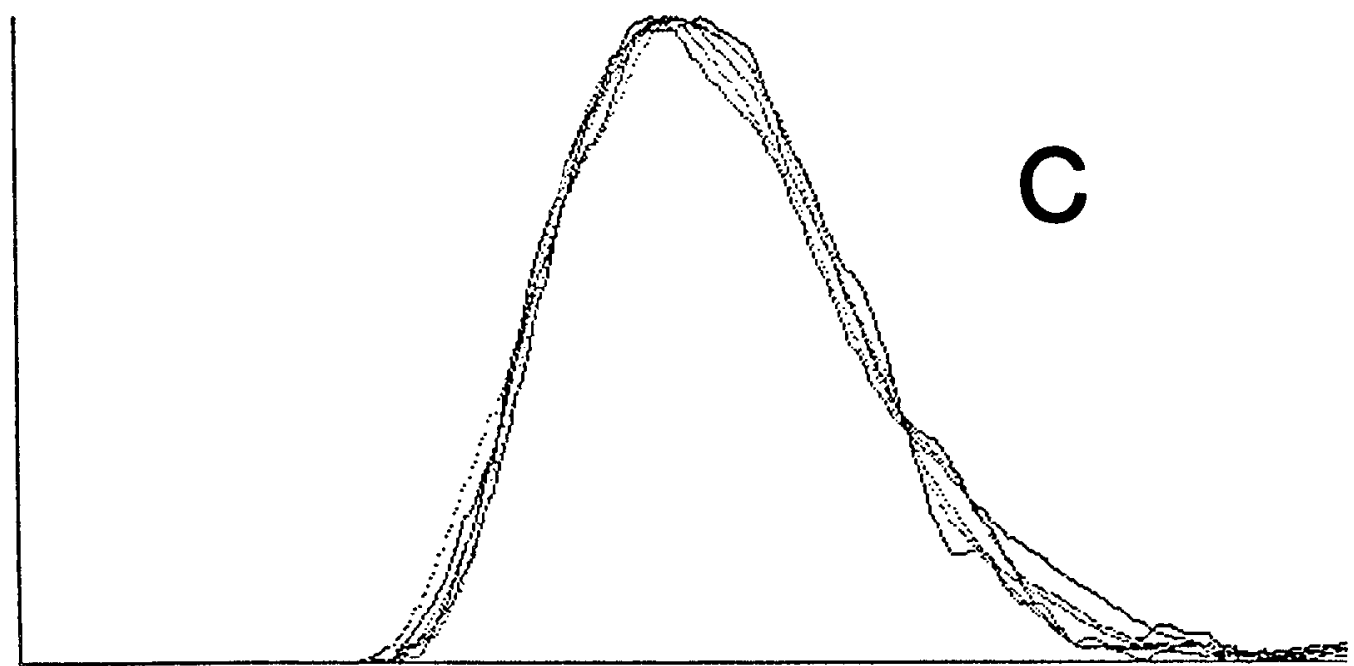

Figure 3. Normalization of tangential velocity profiles. $A, A$ set of typical tangential velocity profiles of the wrist LED, derived from six unloaded movements at slow speeds between targets 3 and $7 . B$, Normalized tangential velocity profiles indicating substantial overlap. The units for the normalized tangential velocity profile have no physical meaning and are therefore not indicated. $C$, Realigned profiles through minimization of the similarity measure $w$ with respect to the averaaed velocitv profile. 

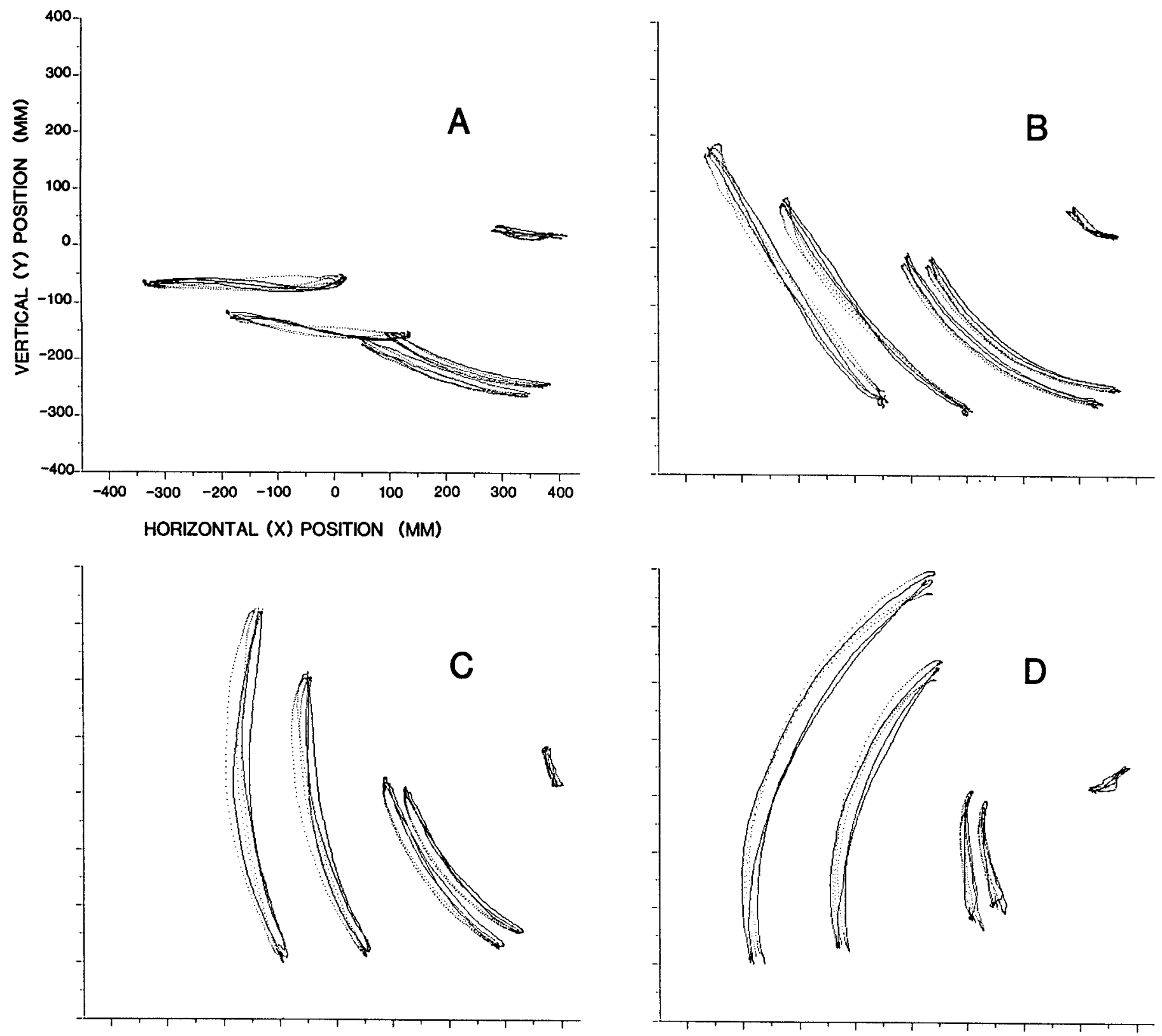

Figure 4. Typical arm trajectories. One subject's medium speed unloaded trajectories between each pair of targets: $A$ 1-5; $B, 2-6 ; C, 3-7 ;$ and $D$ 4-8. In $B-D$ upward movements are dotted lines and downward movements are solid lines; $A$ movements to the left are dotted and movements to the right are solid lines. In $A-B$ the wrist and finger movement paths are approximately straight whereas in $C-D$ the wrist and finger paths are curved.

$$
v^{\prime \prime}(t)=c^{\prime} v^{\prime}\left(c^{\prime} t\right)=c v\left(\frac{c}{a} t\right)
$$

An example of the results of normalization is given in Figure 3, where Figure $3 A$ contains the tangential velocity profiles before normalization and Figure $3 B$ shows the profiles after normalization.

Invariance measures. To determine the degree of similarity of tangential velocity profiles, a measure is composed from intersection and union of an experimental profile aligned with a reference profile. Let $A$ and $B$ be the areas under the experimental and reference profiles, respectively. Then $A \cup$ $B$ is the total area contained beneath both curves, and $A \cap B$ is only the area common to both curves. Then the measure of similarity $w$ is defined as

$$
w=\frac{A \cup B-A \cap B}{B}
$$

Because of ambiguities in defining the beginning and end of an experimental velocity profile, these calculations were confined between beginning and end points defined as $10 \%$ maximum velocity along a profile. Changing the cutoff by a few percent did not appreciably affect the results.

Since the profile shapes are being compared, the fairest measure of similarity would be an alignment of the two curves that minimizes $w$ rather than an alignment at the maximum velocity, which is subject to irregularities in the profiles. For example, the profiles in tigure $3 B$ were averaged to define a reference profile and then were realigned relative to this profile in Figure $3 C$ by minimizing $w$. The result is a considerable improvement in apparent overlap.

Under "Results," it will be seen that paths between two targets are somewhat more curved than are the other paths. To assist in quantifying this difference in curvature, we propose a measure of linearity $l=a / b$ where $a$ is the maximum normal distance from the path to a straight line joining start and end points and $b$ is this straight-line distance. If $I=0$, the path is a perfect straight line; if $I=1$, the path is closer to circular. A number of other linearity measures were tried, but most, such as arc length, were not sufficiently sensitive to curvature. The current measure is simple and scale independent.

\section{Results}

In examining trajectories of the fingertip and the wrist LED, it was observed that there was greater regularity in the wrist trajectories. 


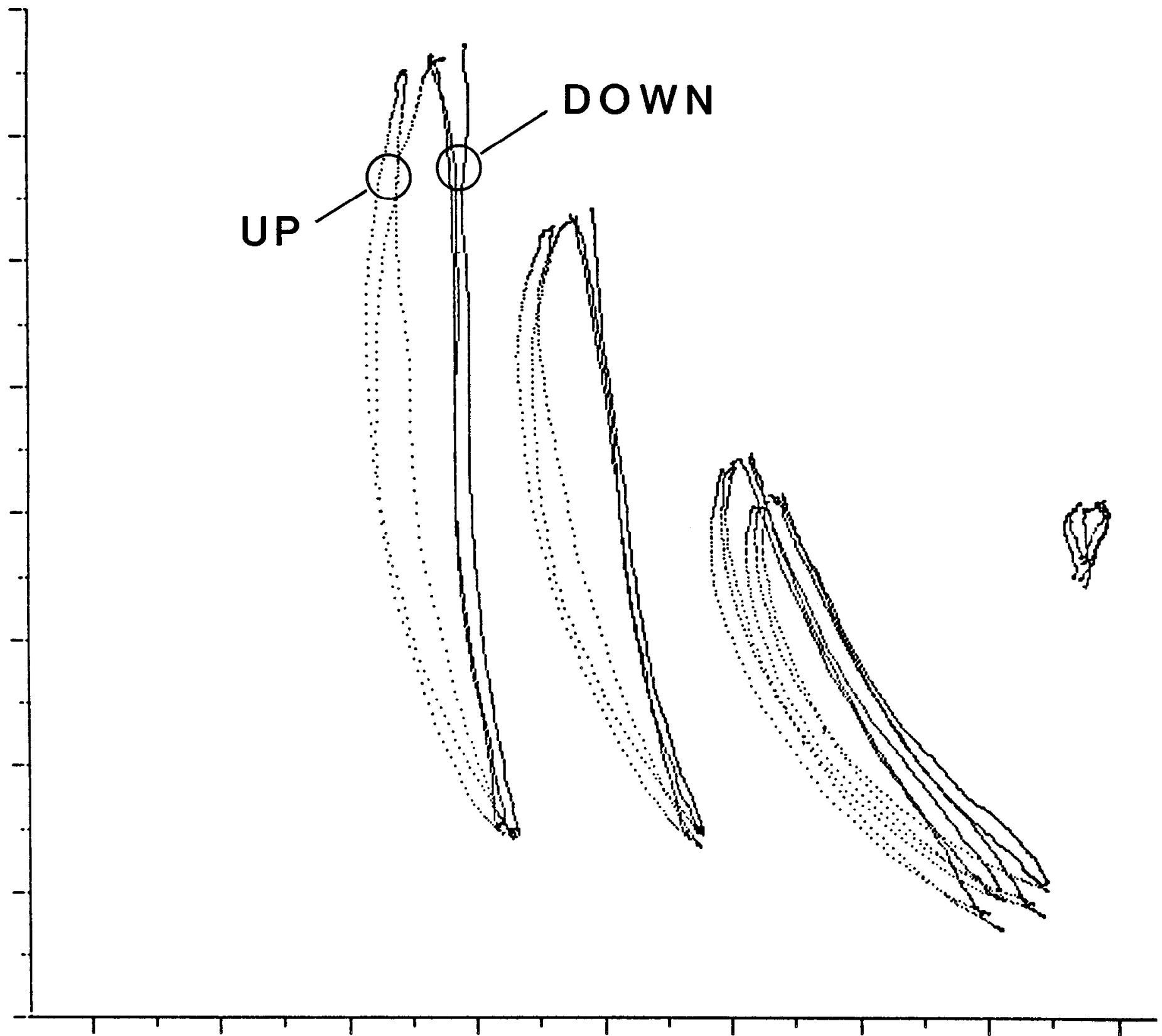

Figure 5. Path dependence on direction. One subject's normal speed, unloaded trajectories between targets 3 and 7 show a dependence on movement direction. Upward movements are dotted lines; downward movements are solid lines. These movements are grouped according to their direction and do not overlap.

The wrist was not constrained in our experiments, and there was some decoupling between wrist movement and arm movement. Similar results were obtained by Lacquaniti and Soechting (1982). Therefore, the results on path shape and tangential velocity profile are presented below in terms of the wrist point.

Path curvature and workspace. Results obtained from previous planar two-joint horizontal arm movement studies suggest that the wrist paths tend to be straight lines (Georgopoulos et al., 1981; Morasso, 1981; Abend et al., 1982; Hollerbach and Flash, 1982). In contrast, we have observed curved as well as straight movements where the amount of curvature is dependent on the workspace. To depict these movements, they are projected into the vertical plane and plotted in Figure 4. The out of plane component was relatively insignificant for fingertip and wrist although, as mentioned previously, the elbow point typically moved substantially out of the vertical plane. We should note that this vertical plane is aligned at $45^{\circ}$ with each camera axis, and the full three-dimensional coordinates of each LED must be constructed.
Substantially straight paths were observed for movements between targets $1-5$ and 2-6 (Fig. 4, $A$ and $B$ ), with linearity measures and standard deviations $I=0.0525 \pm 0.0224$ and $0.0661 \pm 0.0247$, respectively. But the vertical movements between targets 3-7 (Fig. 4C) and particularly movements between targets 4-8 (Fig. 4D) were more curved, with linearity measures $0.0839 \pm 0.0361$ and $0.129 \pm$ 0.077 , respectively. Although these results represent a normal-speed motion without weights for one subject, they generalize to other subjects and to other speed/weight conditions. For two of the five subjects there were marked differences in the paths for upward versus downward movements (Fig. 5). This path dependence on direction has not been observed in previous experiments. Previous studies of vertical movements did not compare movements in opposite directions (Soechting and Lacquaniti, 1981; Lacquaniti and Soechting, 1982); moreover, their movement directions only corresponded to those which in our studies also showed straight-line trajectories. 

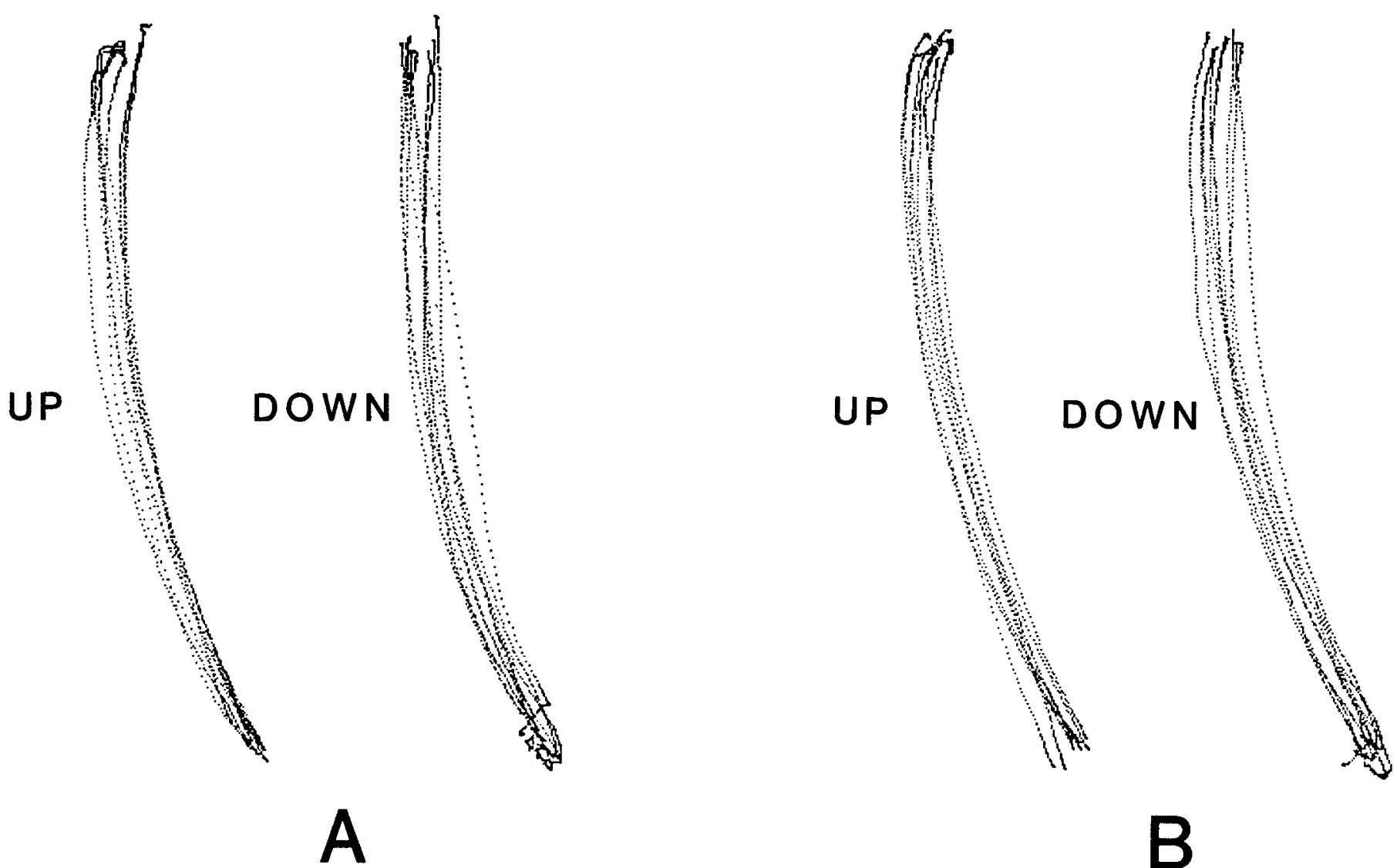

Figure 6. Path invariance across speed and load. A comparison of one subject's wrist trajectories between targets 3 and 7 indicates substantial overlap and no grouping based on speed or load condition. $A$, Six slow movements (three upward and three downward), six medium, and six fast. Upward wrist movements are superimposed on the left; downward wrist movements are superimposed on the right. $B$, Medium speed trajectories for six unloaded movements (again three upward and three downward), six with a 2-lb. hand-held load, and six with a 4-lb. hand-held load.

Although the aim of the current study was not directed toward the effect of practice, we did not actually observe such an effect. The literature indicates that movements without spatial accuracy constraints tend not to show practice effects (Sheridan, 1979). Since the target easily falls within the variability of the gross movement, it is also the case that vision is not needed for the task (Wallace and Newell, 1983).

Path invariance with speed and load. Paths for different movement speeds are compared in Figure $6 A$, which represents overplots of movements between targets 3 and 7 at slow, normal, and fast speeds. Paths for different load weights are compared in Figure $6 B$, representing medium-speed movement without load, with $2-\mathrm{tb}$. load, and with $4-\mathrm{lb}$. load. It can be seen that the trajectory of the wrist point is similar for the different movement speed and load conditions. This observation holds for paths in other parts of the workspace as well.

Scaling of the tangential velocity profile. Tangential velocity profiles were normalized and realigned according to the procedure under "Methods," and compared across movement speeds, load conditions, paths, and even subjects. Vertical movements for one subject are compared at slow, normal, and fast speeds without load in Figure $7 A$; tangential velocities for the downward movements have been made negative to distinguish them from the upward movements. The realignment is defined with respect to the average profile for the medium-speed movement in Figure $4 C$; Figure $7, B$ to $D$, is also realigned to this profile. The average measures of similarity $w$ with standard deviation for the slow, normal, and fast speeds were $0.056 \pm 0.030,0.034 \pm 0.013$, and $0.049 \pm 0.013$ respectively.

Normal-speed movements for the same subject with no load and with a 4-lb. load are compared in Figure $7 B$; the average measure of similarity for the $4-\mathrm{lb}$ movement is $0.040 \pm 0.012$. The load scaling invariance holds for other movement speeds and across movement speeds. Profiles trom tour ditterent target pairs executed under conditions of normal speed and no load are compared in Figure $7 C$, with average similarity measures for movements corresponding to Figure 4, $A-B$ and Figure $4 D$ of $0.056 \pm 0.016,0.052 \pm 0.017$, and $0.065 \pm 0.018$, respectively. Profiles from three different subjects executing fast unloaded movements between targets 3 and 7 are compared in Figure 7D, with similarity measures $0.049 \pm 0.013$ (same movement as before), $0.055 \pm 0.022$, and $0.057 \pm 0.031$, respectively.

The tangential velocity profiles thus overlap substantially across conditions of speed, load, path, and subject. This extends the results of Hollerbach and Flash (1982), who reported speed invariances for restricted horizontal planar arm movements. With respect to the mathematical form of these curves, the reference profile in Figure 7 , $A$ to $D$, is compared to a minimum-jerk profile in Figure $7 E$. The profiles are quite similar $(w=0.052)$ except for the end portions; particularly for the tail end, the averaged experimental profile slopes more gently to zero. It is a consistent result across many experimental profiles that the ends slope more gently than for a minimum-jerk profile; for this reason, using the minimum jerk profile as reference always gives worse results for the invariance measure $w$. Although the particular reference profile in Figure $7 E$ indicates a slight asymmetry, in general there was no consistent trend as to whether the tail portion or the front portion slopes more gently or whether they are roughly comparable.

\section{Discussion}

Taken together, shape invariance for path and tangential velocity profile indicates that subjects execute only one form of trajectory 

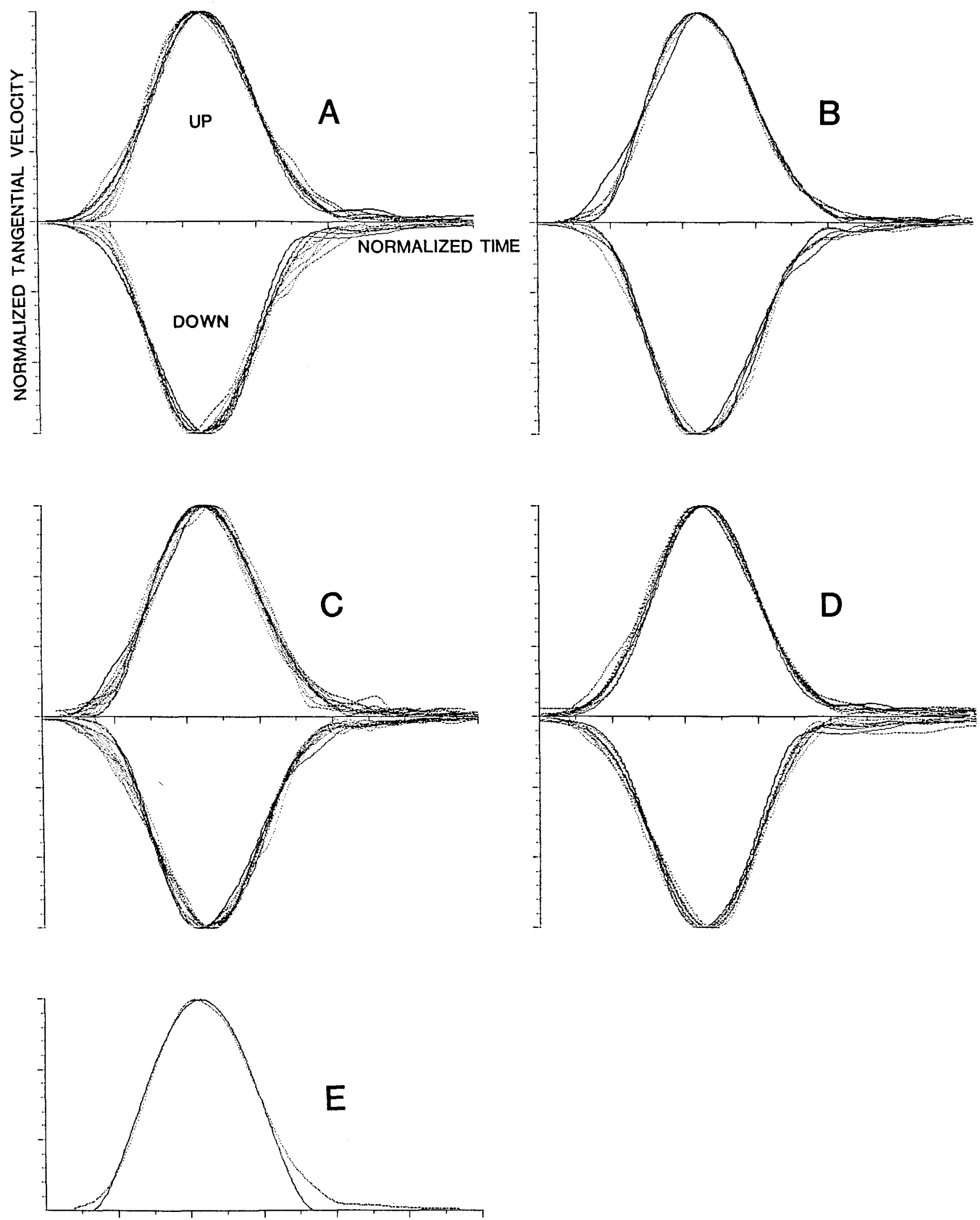

Figure 7. Tangential velocity profile shape invariance across different conditions. A, Different speeds. One subject's unloaded movements between targets 3 and 7 at slow, medium, and fast speeds are shown. Slow and fast movements are dotted lines here, whereas medium-speed movements are presented as solid lines. B. Different loads. One subject's medium-speed movements between targets 3 and 7 were executed unloaded (solid lines) and loaded by a $4-\mathrm{lb}$. hand-held load (dotted lines). $C$, Different movements. One subject's medium-speed unloaded movements between all target pairs are shown. Movements between targets 3 and 7 are shown as solid lines, and those between targets $1-5,2-6$, and $4-8$ are shown as dotted lines. D, Different subjects. Fast speed unloaded movements between targets 3 and 7 from three subjects. Movements of the same subject as presented in $A-C$ are shown as solid lines; movements of the other two subjects are shown as dotted lines. $E$, Comparison of reference profile (dotted lines) to minimum-jerk profile (solid line). 
between any two targets when not instructed to do otherwise. The only changes in the trajectory are simple scaling operations to accommodate different speeds. Furthermore, subjects use the same tangential velocity profile shape to make radically different movements, even when the shapes of the paths are not the same in extrinsic coordinates. Different subjects use the same tangential velocily prolile shape.

One reason for these experiments was to infer the effect of gravity on trajectory shape when movement speed is changed. The hy pothesis was that if path and tangential velocity profile were invariant, then this would be consistent with a simplifying strategy for joint torque formation by separation of gravity torques from dynamic torques and a uniform scaling of the tangential velocity profile (Hollerbach and Flash, 1982). The affirmative results presented here are a sterner test than those in Hollerbach and Flash (1982) because of the presence of gravity. In contrast, tangential velocity profile irvariance with load is a new result and is not accounted for by the dynamic time scaling property. Later we show that dynamic scaling properties can be extended for load scaling as well as for time scaling to account for this result.

Path shape. The dependence of path curvature on the movement start and end points is a new result and differs from the approximate linearity of horizontal planar movements in previous experiments (Morasso, 1981; Abend et al., 1982; Hollerbach and Flash, 1982). There are three major experimental differences that could account for the curvature in our study. First, gravity acts in the vertical planar movements but not in the horizontal planar movements. Conceivably, curvature could result from difficulties in handling gravity in certain directions or from energy considerations. In two subjects in particular, the upward movements were curved and the downward movements were fairly straight. At the beginning of the upward movement, gravity and shoulder joint acceleration both exert torques at the elbow that tend to cause the elbow to extend. At the beginning of the downward movement the forearm is almost vertical so that there is little gravity torque, and the shoulder acceleration tends to cause the elbow to flex.

Second, the vertical plane movements are in some sense more natural than the horizontal plane movements, since people seldom make the kinds of horizontal movements required by the planar pantograph. Third, the restricted degrees of freedom in the horizontal movements places these movements into the class of compliant motions (Brady et al, 1982), rather than into the class of free arm trajectories as in the present studies. A compliant motion is defined as any trajectory in which the hand motions are constrained by external contact; in the directions of the contact only forces can be generated and not displacements. At least in robotics, compliant motions are executed under a different control strategy from free motions, so that perhaps the compliant horizontal-planar movements are also fashioned with a different control strategy. In vertical movements it would be as if the motions had to be in contact with a vertical wall the whole time. We plan to conduct experiments to ascertain more precisely where the different experimental results arise from.

Wo do not yct have a full explanation for why certain movements are curved whereas others are straight. Joint limits do not seem to be the explanation, since movements were curved in intermediate regions. Preliminary analysis indicates that the vertical movements are generated by movement of the shoulder joint only, so that naturally the hand trajectories are curved. For the upward and diagonally inward curved motion between targets 4 and 8 , both the elbow and shoulder joints seem to be moving. Hollerbach and Atkeson (1984) have shown that joint-interpolated motions lead to well defined curved motions, but whether the target 4-8 movements can be explained in terms of joint interpolation is not yet known.

Dynamic scaling properties for speed and load. A partial explanation for the velocity profile invariances with speed and load may be provided by an examination of arm movement dynamics. Holler- bach and Flash (1982) suggested that movement speed could be simply scaled if the joint torques due to gravity $\tau_{\text {gravity }}^{\text {arm }}$ are separated from the joint torques arising from the acceleration and velocity product terms $\tau$ drive. Then the total joint torques $\tau$ are (Appendix 1 )

$$
\underline{\tau}(t)=\tau_{\text {orive }}^{\text {am }}(t)+\tau_{\text {gravity }}^{\text {amm }}(t)
$$

where the superscript "arm" is introduced to distinguish the analysis of load dynamics later. If the movement is sped up or slowed down by a factor $c$, the new joint torques $\underline{\tilde{\tau}}$ are related to the old by

$$
\tilde{\underline{z}}(t)=c^{2} \underline{\underline{q}}_{\text {drive }}^{\text {arm }}(c t)+\underline{q}_{\text {gravity }}^{\text {arm }}(c t)
$$

The amplitudes of the drive torques scale by $c^{2}$ whereas the gravity torque amplitudes remain unchanged, which is the reason for the separation of joint torques into two components. Thus, the now movement dynamics are simply obtained from the old.

To understand the effect of load on the underlying dynamics, we hypothesize a phantom arm that carries the load at the tip and mimics the movement of the actual arm (Fig. 8). This phantom arm has massless links and the same link lengths as the actual arm. Again, the phantom arm joint torques $\tau^{\text {load }}$, where the superscript "load" is used to indicate torques associated with the phantom arm, are divided into drive and gravity components.

$$
\tau^{\text {load }}=\tau_{\text {drive }}^{\text {load }}+\tau_{\text {gravity }}^{\text {load }}
$$

If the load mass scales by $r$, and under the assumption that the load moment of inertia scales by $r$ as well, the new phantom arm torques $\tilde{\tau}^{\text {load }}$ are (Appendix II)

$$
\tilde{\tau}^{\text {load }}=r \tau_{\text {drive }}^{\text {logad }}+r \underline{\tau}_{\text {gravity }}^{\text {load }}
$$

If speed scaling by a factor $c$ is included as well, then from equation B 16 (Appendix II)

$$
\underline{\tilde{\tau}}^{\text {load }}=c^{2} r \underline{\tau}_{\text {drive }}^{\text {load }}+r{\underline{\tau_{\text {gravity }}}}_{\text {load }}^{\text {gad }}
$$

The phantom arm torques are combined with the actual arm torques to indicate how the joint torques vary with speed and load.

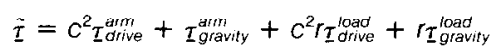

The dynamics for the new movement are obtained by simple linear combination of dynamic components of the old movement. In principle, therefore, it is not difficult for a motor controller to change movement speed and load, provided that the velocity profile is kept the same. If the velocity profile shape were changed, then the movement dynamics would change differently and would have to be refashioned in a more complex manner.

A number of issues remain with regard to these dynamic scaling results. How are the initial torques for the first instance of a movement generated? If the motor controller has the ability to fashion the correct torques for one movement, why does it not use this same ability for all subsequent movements rather than utilize the dynamic scaling properties? Among the possibilities we are considering, the first is a generalized motor tape where only one movement between points need be known if the dynamic components in equation 6 are stored separately. A motor tape for each possible start and finish point would be required, however, which renders this concept still unattractive. A second possibility is a modification of tabular approaches (Raibert, 1978), where the dimensionality and parameter adjustment problem could be reduced by separate tables for the four components in equation 6 . The problem of how the tables are adapted for new, sudden loads is now solved, and the higher velocity and acceleration dimensions of the tables could be eliminated because slower movements could be scaled up. However, the resulting table sizes still required are not clear.

Further experiments are suggested by the assumptions about the linear scaling of the load moments of inertias. We would predict that there would be some trajectory deviation if the load moment of inertia scaling departs substantially from linearity from trial to trial. It should be mentioned that a complete proof of trajectory scaling 


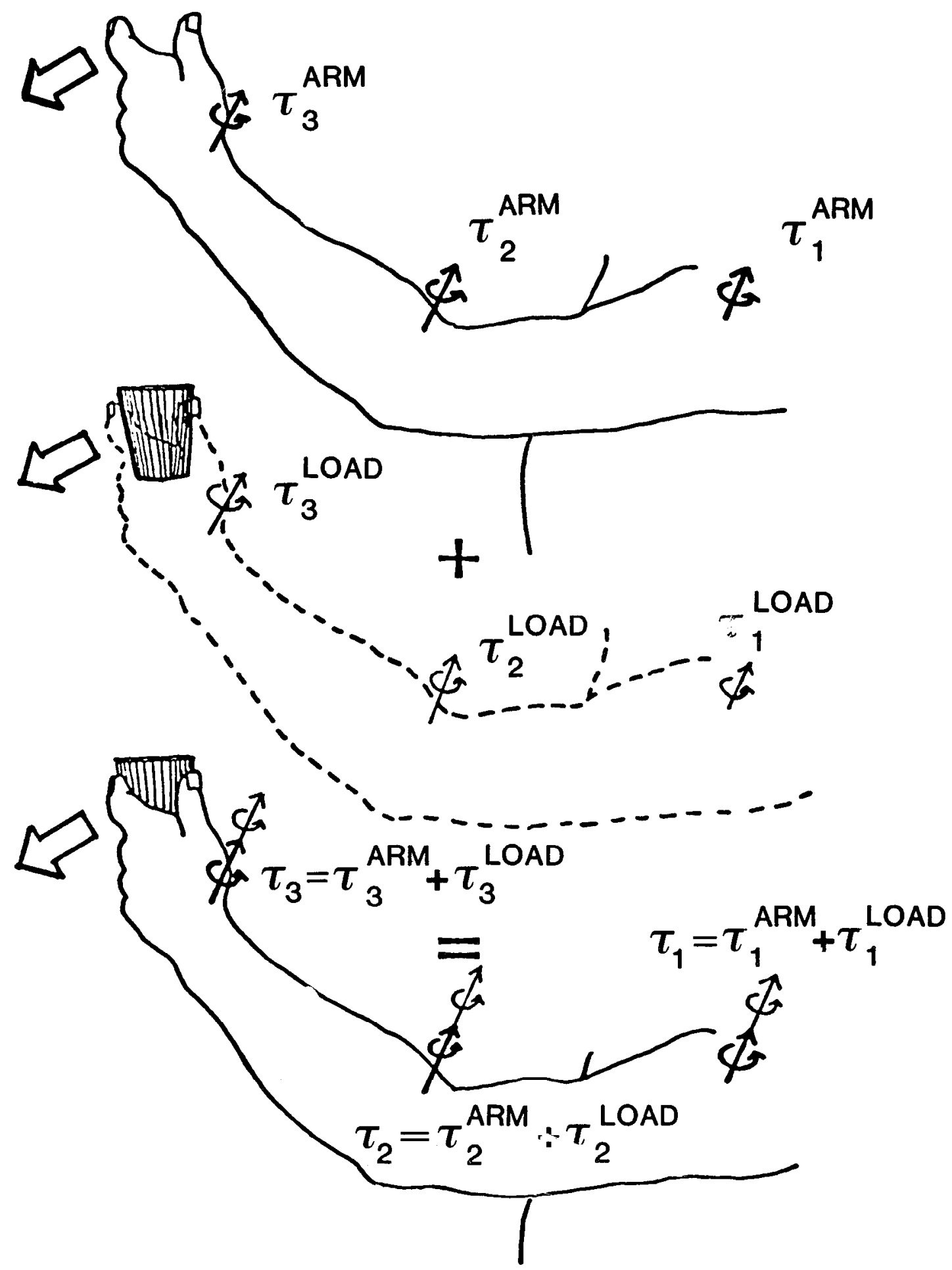

Figure 8. Separation of arm dynamics and load dynamics computation. For any movement the torques necessary to drive the arm ( $\tau^{\text {amm}}$ ) without the load (top arm) can be combined with the torques necessary to drive the load. The load torques ( $\left.\underline{\tau}^{\text {load}}\right)$ can be found by examining a phantom arm (dashed arm) that mimics the geometrical structure of the actual arm but is massless, so that it requires no torques to drive it. The phantom arm does require torques $\left(\tau^{\text {load }}\right)$ to drive the load it holds at the tip. The total torques necessary to drive the arm plus the load are the sum of $\left(\tau^{\text {load }}\right)$ and $\left(\tau^{\text {arm }}\right)($ bottom arm $)$.

would require the comparison of accelerations as well as of velocities, because similarly shaped velocity profiles might have fairly different acceleration profiles. The accuracy of our data does not currently warrant the calculation of accelerations, although future development of our measurement system will strive for improved accuracy. For the more accurate measurements of horizontal movements with the planar pantograph, the acceleration and torque scaling were seen to hold reasonably well (Hollerbach and Flash, 1982).

Tangential velocity profile shape. The dynamic scaling properties do not imply anything about the shape of the velocity profile for a given movement between two points, only that once chosen the motor controller should retain it under speed and load changes. This result also does not require that movements between different points 
have the same velocity profile. It is beyond the scope of the current studies to explain why the velocity profile has its particular shape and why this shape is maintained across different paths.

In examining possible hypotheses for trajectory shape, the curved paths observed in our studies are in conflict with the model of minimum jerk in terms of task coordinates (Flash, 1983), which predicts only straight lines for unconstrained movement, whereas the velocity profiles are consistent with the minimum jerk model. The other explanations for velocity profile shape mentioned in the introduction were formulated for single-joint movement and do not pertain directly to multi-joint movement. Whether these explanations can be easily extended to the multi-joint case is an open question, and the explanation for trajectory shape must still be considered unresolved.

\section{Appendix I}

The change of movement dynamics with uniform speed scaling by a factor $c$ was presented in (Hollerbach, 1984) but tor reterence is rederived below. Let $\underline{\theta}(t)=\left(\theta_{1}(t), \theta_{2}(t), \ldots, \theta_{n}(t)\right)$ represent the time-varying $n$-dimensional vector of joint angles and $\underline{\tau}(t)=\left(\tau_{1}(t)\right.$, $\left.\tau_{2}(t), \ldots, \tau_{n}(t)\right)$, the corresponding time-varying vector of joint torques. A new trajectory $\tilde{\theta}(t)=\underline{\theta}(c t)$ is defined that compresses or expands the time axis uniformly by a factor $c$. If $c>1$, the movement is sped up; if $c<1$, the movement is slowed down. The joint velocities and accelerations are related to the old as follows.

$$
\begin{aligned}
& \dot{\hat{\theta}}(t)=c \underline{\theta}(c t) \\
& \ddot{\tilde{\theta}}(t)=c^{2} \ddot{\theta}(c t)
\end{aligned}
$$

The movement dynamics can be compactly represented as

$$
\underline{\tau}(t)=I(\underline{\theta}(t)) \underline{\theta}(t)+\underline{\dot{\theta}}(t) \cdot C(\underline{\theta}(t)) \cdot \underline{\dot{\theta}}(t)+g(\underline{\theta}(t))
$$

where $/(\underline{\theta}(t))$ is the $n \times n$ inertia matrix, $C(\underline{\theta}(t))$ is the $n \times n \times n$ matrix of coriolis and centripetal coefficients, and $g(\underline{\theta}(t))$ is the $n$ vector of gravity torques. The new torques $\tilde{\tau}$ for the time-scaled movement are obtained by substituting equation $\mathrm{A} 1-\mathrm{A} 2$ into equation A3:

$$
\underline{\tilde{\tau}}(t)=c^{2}[/(\underline{\theta}(c t)) \ddot{\theta}(c t)+\underline{\dot{\theta}}(c t) \cdot C(\underline{\theta}(c t)) \cdot \underline{\dot{\theta}}(c t)]+g(\underline{\theta}(c t))
$$

This expression can be more compactly represented by rewriting the dynamics in terms of drive torques $\tau_{\text {drive }}$ and gravity torques $\tau_{\text {gravity. }}$.

$$
\underline{\tau}(t)=\tau_{\text {drive }}(t)+\underline{I}_{\text {gravity }}(t)
$$

where

$$
\begin{aligned}
\underline{\tau}_{\text {drive }}(t) & =l(\underline{\theta}(t)) \underline{\ddot{\theta}}(t)+\underline{\dot{\theta}}(t) \cdot C(\underline{\theta}(t)) \cdot \underline{\dot{\theta}}(t) \\
\tau_{\text {gravity }}(t) & =g(\underline{\theta}(t))
\end{aligned}
$$

Then equation A4 becomes

$$
\tilde{\tilde{\tau}}(t)=c^{2} \underline{\tau}_{\text {drive }}(c t)+\underline{\tau}_{\text {gravity }}(c t)
$$

\section{Appendix II}

The change of movement dynamics with load is best demonstrated by combining the dynamics of the arm without a load with the dynamics of a massless phantom arm carrying the load. We consider here the dynamics of the massless arm driving the load. Due to its movement, the load exerts a force $f$ and a torque $n$ on the tip of the phantom arm given by the Newton-Euler equations

$$
\begin{aligned}
& f=m \ddot{r} \\
& n=l \underline{\dot{\omega}}+\underline{\omega} \times l \underline{\omega}
\end{aligned}
$$

where $m$ is the load mass, $r$ is the acceleration of the load center of gravity, $I$ is the inertia tensor of the load about the center of gravity, and $\underline{\omega}$ and $\underline{\dot{\omega}}$ represent the angular velocity and acceleration of the loads. Again, the gravity torques will be treated separately later.

How can we turn forces and torques at the tip of the phantom arm into joint torques? Let $x=\left(x_{1}, x_{2}, \ldots, x_{m}\right)$ represent the $m$ dimensional position and orientation vector that locates the load in space. Then the load trajectory is related to the joint trajectory by the vector function $h=\left(h_{1}, h_{2}, \ldots, h_{m}\right)$ where

$$
x=h(\underline{\theta})
$$

Differentiating,

$$
\dot{x}=J \underline{\theta}
$$

where elements of the Jacobian matrix $J$ are given by

$$
J_{i j}=\frac{\partial h_{i}(\underline{\theta})}{\partial \theta_{i}}
$$

By the principle of virtual work (Brady et al., 1982),

$$
\underline{I}_{\text {drive }}^{T} \dot{\theta}=\left[f^{T} n^{T}\right] \dot{x}
$$

where $\tau_{\text {drive }}^{T}$ is the drive torque for the phantom arm. Substituting from equation B4 and transposing, it can be seen that

$$
\tau_{\text {drive }}=J\left[\begin{array}{l}
f \\
n
\end{array}\right]
$$

We have found the torques necessary to drive the phantom arm with the load at the tip. We calculate the torques necessary to hold the load up against the force of gravity in the same way:

$$
\tau_{\text {graviy }}=J^{\prime}\left[\begin{array}{c}
m g \\
0
\end{array}\right]
$$

where $m$ is the mass of the object and $g$ is the gravitational constant.

Suppose the load mass and moment of inertia scale by a factor $r$. Then the new mass $\dot{m}$ and inertia $\tilde{I}$ are linearly related to the old by

$$
\begin{gathered}
\tilde{m}=r m \\
i=r
\end{gathered}
$$

The inertia scaling is a restrictive assumption that is not generally true, since a nonuniform change in load shape or density would change the inertia tensor in a more complex manner. The assumption of linear inertia scaling is the simplest case and may suggest experiments which are discussed in the main text. Then the new load force and torque are obtained from the Newton-Euler equations as

$$
\begin{aligned}
& \tilde{f}=r f \\
& \tilde{n}=r n
\end{aligned}
$$

Note that if the moment of inertia did not scale linearly, then the load force and torque would change differently. The new drive torques for the phantom arm are obtained from equation $\mathrm{B} 7$ and equation B11-B12:

$$
\underline{\underline{I}}_{\text {drive }}=r_{\underline{\text { drive }}}
$$

The gravity torques for the phantom arm scale by $r$ as well since they are proportional to the load mass.

$$
\tilde{\underline{\tau}}_{\text {gravity }}=r \underline{\tau}_{\text {gravity }}
$$

Combining equations $\mathrm{B} 13$ and B14, the load-scaled total phantom arm torques are

$$
\tilde{\tau}=r_{\text {drive }}+r \underline{\tau}_{\text {gravity }}
$$

When speed scaling of the phantom arm is considered as well, the drive and gravity torques for the phantom arm scale differently. From equation $\mathrm{B} 1-\mathrm{B} 2$, the net force and torque scale by $\mathrm{c}^{2}$, hence the drive torques scale by $c^{2}$ from equation $B 7$, while again the gravity torques are speed independent:

$$
\underline{\tilde{i}}=c^{2} r \underline{\tau}_{\text {drive }}+r \underline{\tau}_{\text {gravity }}
$$


The joint torques of the phantom arm driving the load are simply added to the joint torques necessary to drive the arm with no load along the same trajectory by the principle of superposition.

\section{References}

Abend, W., E. Bizzi, and P. Morasso (1982) Human arm trajectory formation. Brain 105: 331-348.

Andriacchi, T. P., S. J. Hampton, A. B. Schultz, and J. O. Galante (1979) Three-dimensional coordinate data processing in human motion analysis. J. Biomech. Eng. 101: 279-283.

Annet, J., C. W. Golby, and H. Kay (1958) The measurement of elements of an assembly task - the information output of the human motor system. J. Exp. Psychol. 10: 1-11.

Antonsson, E. K. (1978) The derivation and implementation of a dynamic three-dimensional linkage analysis technique. S.M. dissertation, Massachusetts Institute of Technology, Department of Mechanical Engineering, Cambridge, MA.

Antonsson, E. K. (1982) A three-dimensional kinematic acquisition and intersegmental dynamic analysis system for human motion. Ph.D. dissertation, Massachusetts Institute of Technology, Department of Mechanical Engineering, Cambridge, MA.

Beggs, W. D. A., and C. I. Howarth (1972) The movement of the hand towards a target. Q. J. Exp. Psychol. 24: 448-453.

Brady, J. M., J. M. Hollerbach, T. L. Johnson, T. Lozano-Perez, and M. T. Mason (1982) Robot Motion: Planning and Control, MIT Press, Cambridge, MA.

Carlton, L. G. (1980) Movement control characteristics of aiming responses. Ergonomics 11: 1019-1032.

Conati, F. C. (1977) Real-time measurement of three-dimensional multiple rigid body motion. S.M. Dissertation, Massachusetts Institute of Technology, Department of Mechanical Engineering, Cambridge, MA.

Crossman, E. R. F. W. and P. J. Goodeve (1983) Feedback control of handmovement and Fitts' Laws. (Paper presented at the meeting of the Experimental Psychological Society, Oxford, July 1963.) Q. J. Exp. Psychol. 35A: 251-278.

Flash, T. (1983) Organizing principles underlying the formation of hand trajectories. Ph.D. dissertation, Harvard/MIT Division of Health Sciences and Technology, Cambridge, MA.

Georgopoulos, A. P., J. F. Kalaska, and J. T. Massey (1981) Spatial trajectories and reaction times of aimed movements: Effects of practice, uncertainty, and change in target location. J. Neurophysiol. 46: 725-743.

Hogan, N. (1984) An organizing principle for a class of voluntary movements. J. Neurosci. 4: 2745-2754.

Hollerbach, J. M. (1981) An oscillation theory of handwriting. Biol. Cybern. 39: $139-156$.

Hollerbach, J. M. (1982) Computers, brains, and the control of movement. Trends Neurosci. 5: 189-192.

Hollerbach, J. M. (1984) Dynamic scaling of manipulator trajectories. ASME J. Dynamic Syst. Meas. Control 106: 102-106.

Hollerbach, J. M., and C. G. Atkeson (1984) Characterization of jointinterpolated arm movements. Soc. Neurosci. Abstr. 10: 338.

Hollerbach, J. M., and T. Flash (1982) Dynamic interactions between limb segments during planar arm movement. Biol. Cybern. 44: 67-77.

Jagacinski, R. J., E. J. Hartzeil, S. Ward, and K. Bishop (1978) Fitts' law as a lunction of system dynamics and largel uncerlainty. J. Molor Behav. 10: 123-131.

Jagacinski, R. J., D. W. Repperger, M. S. Moran, S. L. War, and B. Glass (1980) Fitts' law and the microstructure of rapid discrete movements. J. Exp. Physiol. Hum. Percept. 6: 309-320.
Keele, S. W. (1968) Movement control in skilled motor performance. Psychol. Bull. 70: 127-134.

Lacquaniti, F., and J. F. Soechting (1982) Coordination of arm and wrist motion during a reaching task. J. Neurosci. 2: 399-408.

Lacquaniti, F., J. F. Soechting, and C. A. Terzuolo (1982) Some factors pertinent to the organization and control of arm movements. Brain Res. 252: 394-397.

Langolf, G. D., D. B. Chaffin, and J. A. Foulke (1976) An investigation of Fitts' Law using a wide range of movement amplitudes. J. Motor Behav. 8: $113-128$

McClellan, J. H., T. W. Parks, and L. R. Rabiner (1973) A computer program for designing optimum FIR linear phase digital filters. IEEE Trans. Audio Electroacoust. $A \cup$ 21: 506-526.

Meyer, D. J. E. K. Smith, and C. Wright (1982) Models for the speed and accuracy of aimed movements. Psychol. Rev. 89: 449-482.

Murassu, P. (1981) Spatial control of arm movements. Exp. Brain Res. 42: 223-227

Morasso, P. (1983) Three dimensional arm trajectories. Biol. Cybern. 48: 18.

Nelson, W. (1983) Physical principles for economies of skilled movements. Biol. Cybern. 46: 135-147.

Raibert, M. H. (1978) A model for sensorimotor control and learning. Biol. Cybern. 29: 29-36.

Reece, D. A. (1981) A Selspot-based data acquisition system for use in a clinical motion study laboratory. M.S. dissertation, Case Western Reserve University, Department of Electrical Engineering and Applied Physics, Cleveland, $\mathrm{OH}$.

Ruitenbeek, J. C. (1984) Invariants in loaded goal directed movements. Biol. Cybern. 51: 11-20.

Schmidt, R. A., H. Zelaznik, B. Hawkins, J. S. Frank, and J. T. Quinn, Jr. (1979) Motor-output variability: A theory for the accuracy of rapid motor acts. Psychol. Rev. 86: 415-451.

Schmidt, R. A., D. E. Sherwood, H. N. Zelaznik, and B. J. Leikind (1984) Speed-accuracy trade-offs in motor behavior: Theories of impulse variability. In The Psychology of Motor Behavior, U. Kleinbeck, H. Heuer, and K. -H. Schmidt, eds., Springer-Verlag, Berlin, in press

Sheridan, M. R. (1979) A reappraisal of Fitts' Law. J. Motor Behav. 11: 179188.

Soechting, J. F. (1984) Effect of target size on spatial and temporal charac teristics of a pointing movement in man. Exp. Brain Res. 54: 121-132.

Soechting, J. F., and F. Lacquaniti (1981) Invariant characteristics of a pointing movement in man. J. Neurosci. 1: 710-720.

Soechting, J. F., and F. Lacquaniti (1983) Modification of trajectory of a pointing movement in response to a change in target location. J. Neurophysiol. 49: 548-564.

Stein, R. B., M. N. Oguztoreli, and C. Capaday (1985) What is optimized in muscular movements? In Human Muscle Performance: Factors Underlying Maximal Performance, N. McCartney et al., eds., Kinetic Publishers, New York, in press.

Taylor, F. V., and H. P. Birmingham (1948) Studies of tracking behavior. II. The acceleration pattern of quick manual corrective responses. J. Exp. Psychol. 38: 783-795.

Tetewsky, A. K. (1978) Implementing a real time computation and display algorithm for the Selspot system. S.M. dissertation, Massachusetts Institute of Technology, Department of Electrical Engineering, Cambridge, MA.

Viviani, P., and C. Terzuolo (1982) Trajectory determines movement dynamics. Neuroscience 7: 431-437.

Wallace, S. A., and K. M. Newell (1983) Visual control of discrete aiming movements. Q. J. Exp. Psychol. 35A: 311-321. 